\title{
So optimieren Sie die Zusammenarbeit
}

\author{
Qualitätsmanagement hilft bei der Teambildung Lob und Zuspruch der \\ Klientinnen und gelingende Projekte mit Strahlkraft über die Region hinaus \\ bringen ein Team weiter und halten es zusammen. Wenn jedoch nach der \\ Euphorie der Gründung die Gruppe in eine Phase der Teambildung eingetreten \\ ist, müssen neue Impulse her. Setzen Sie daher auf Qualität - auch wenn dies \\ Zeit erfordert. \\ Gabriele Stenz
}

\section{$\mathbf{S}$} tellen Sie sich vor, Ihr Schöpfungsprozess der gemeinsamen Arbeit in einer Hebammenpraxis ist schon weit gediehen. Ihre Visionen von wunderschönen, hellen, bezahlbaren Räumen und vielen kompetenten Kolleginnen, mit den Sie gedeihlich zusammenarbeiten, ist endlich Wirklichkeit geworden. Die Hebammen bieten in den neuen Räumen ein breites Spektrum für Schwangere, Mütter, Eltern, Babys an. Sie schwärmen täglich zu den Wochenbetten aus, besprechen sich häufig und vertreten sich zuverlässig bei Bedarf. Doch seit einiger Zeit bemerken Sie, dass die organisatorische Arbeit immer an den gleichen Kolleginnen hängen bleibt und sich zu den Besprechungen immer mehr Hebammen entschuldigen als anwesend sind. Eine gewisse Entscheidungsmüdigkeit hat sich breitgemacht, keine will mehr so recht Verantwortung übernehmen, familiäre Verpflichtungen stehen im Vordergrund, nichts ist mehr selbstverständlich.

\section{Was will die „Kundin“ wirklich?}

Neue Impulse und nachhaltige Motivation entstehen durch die gemeinsame Freude am Erfolg. Lob und Zuspruch der Klientinnen und gelingende Projekte mit Strahlkraft über Ihre Region hinaus bringen ein Team weiter und halten es zusammen. Um dort hinzugelangen, gilt es, auf Qualität zu setzten und damit die Zufriedenheit der Frauen in den Mittelpunkt zu stellen.

Qualität ist ein weitgefasster Begriff. Das ständige Streben nach der vermeintlichen Vollkommenheit beinhaltet nicht nur die originäre Hebammenarbeit, sondern alle sogenannten Wertschöpfungsprozesse, die Arbeitsbedingungen, die Mitarbeiterinnenzufriedenheit und das Umfeld der Hebammenpraxis. Eben das ganze Drumherum, welches unverzichtbar für die Erbringung der eigentlichen Hebammenarbeit ist. Das Denken an Qualität muss zum umfassenden systematischen Prozess einer Teamkultur werden, die alle Beteiligten durchdringt. Und das braucht Zeit und eine gewisse Systematik,

\section{Qualitätsziele mit dem Team und den Klientinnen finden}

Als Team sollten Sie über eine Idee, einen guten Plan verfügen, mit dem es Ihnen gelingt Ihr Hebammendasein lange und begeistert zu leben. „Wer nicht weiß, in welchen Hafen er segeln will, für den ist kein Wind der richtige“, so formulierte es der Philosoph Seneca bereits vor fast 2.000 Jahren. Er meinte damit die Lebensziele, die uns erstrebenswert erscheinen. Hebammen haben bekanntlich unterschiedliche Ziele oder Wegweiser zum beruflichen Glück.

Für ein Hebammenteam gibt es die unternehmerischen Ziele. Diese zu finden, zu vereinbaren, als gemeinsames Projekt zu verfolgen, das bringt neuen Schwung in die gute Sache. Den meisten Hebammen sind die unternehmerischen Elemente in ihrer Arbeit unangenehm, deshalb: Fangen Sie es spielerisch an, werden Sie sich der gemeinsamen Vision bewusst. Daraus werden mit der Zeit strategische Ziele und mit wachsender Erfahrung leiten Sie daraus die Mittel und Methoden zur Erreichung der „Praxisziele ab. Diese „Qualitätspolitik“ ist die Ausgangsbasis für alle grundsätzlichen Überlegungen. Bevor Sie loslegen, klären Sie:

- Wer sind wir?

- Was wollen wir?

- Wo wollen wir hin?

- Wie erreichen wir das?

Das klingt recht banal, möglicherweise glauben Sie auch, das längst geklärt zu haben. Doch denken Sie an die nachlassende Motivation im Team. Wäre das allen so klar, hätte es kein Motivationsloch gegeben. Wenn Sie Antworten haben, grenzen Sie sie so gut wie möglich ein. Denn nun geht es nicht mehr um das große Ganze, sondern um einzelne und konkrete Arbeitsbereiche. Anhand der Fragen nehmen Sie jeden einzelnen Arbeitsprozess unter die Lupe:

- Welche Maßnahmen müssen ergriffen werden, damit die Versorgungsqualität der Familien zunimmt?

- Was macht die Frauen zufrieden mit unserer Leistung?

- Brauchen wir neue Geräte, Fortbildungsmaßnahmen, Besprechungsstrukturen? 
- Muss an der Außendarstellung gearbeitet oder die Website geändert werden?

- Welche Veränderungsprozesse kann das Team durchlaufen?

_ Was muss getan werden, damit die Hebammen zufriedener sind und „kundenorientierter" handeln?

Dieses Raster wenden Sie auf jeden, vom Team eingebrachten Arbeitsprozess an. Es ist eine aufwändige Arbeit, die sich lohnt, wenn sie gut dokumentiert wird. Am Ende halten Sie die inhaltliche Struktur für den nun folgenden Teamprozess in den Händen. Das ist Ihr Kursbuch für eine lange Reise mit vielen Abenteuern.

In einem moderierten Workshop errichtet das Team einen Leuchtturm für die Sicherheit. Die gemeinsame Vision für die Hebammenpraxis, ein Leitsatz oder Leitbild entsteht, der in etwa so lautet: „Als überregionale Hebammenpraxis bieten wir den jungen Familien eine sichere (selbstbestimmte) Wochenbettbetreuung (ihre Angebote) und begeistern sie durch Qualität, Sicherheit, Freundlichkeit und Fachkompetenz mit unserem professionellen Team. Wir leben eine kontinuierliche, auf Innovation und Lernen beruhende Qualität der Betreuung". Sollten Sie einmal die Richtung verlieren, mit dem Leitbild wissen Sie wieder, wohin die Reise geht. Teilen Sie Arbeitsbereichsaufgaben und Verantwortlichkeiten im Team auf. Jede hat besondere Fähigkeiten und Talente und bringt sich gerne ein, wenn die Aufgabe genau beschrieben und klar ist. Planen Sie dafür viele Rückmeldeschleifen ein, es klappt nicht immer im ersten Anlauf.

\section{Alle sitzen in einem Boot, doch wohin steuern wir?}

Ohne die Beteiligung aller Hebammen bleibt das Ziel „Qualität“ eine leere Worthülse. Alle müssen von der Sinnhaftigkeit und Notwendigkeit der Vision, der Strategie, den mit der Verbesserung verbundenen Maßnahmen überzeugt sein und sich für sie begeistern. Dies kann schon bei der Visionsentwicklung geschehen, sollte aber spätesten, wenn es um den konkreten Aufgabenbereich einer Hebamme geht, eindeutig sein. Reden Sie miteinander und finden heraus, welche Qualitätsziele die Kollegin mit ihrem Aufgabenbereich verknüpft. Bestehen Sie in den Planungsgesprächen und der Umsetzung auf einer bindenden Selbstverpflichtung und Eigenaktivität der Kollegin. Etablieren Sie eine Lern-Kultur, denn Fehler zu verbergen ist kontraproduktiv. Alle Teammitglieder werden nur dann bereit sein, sich aktiv an der Festlegung der Qualitätsziele zu beteiligen, wenn sie wissen, dass Fehler als wertvoller Ausgangspunkt für Verbesserungs- und Lernprozesse betrachtet werden.

\section{Die Familien sind Ihre Leitsterne}

Wundert es Sie, dass der „Kundenbegriff“ erst jetzt erscheint, nachdem die Bedürfnisse der Teammitglieder im Mittelpunkt standen? Sie müssen zunächst eine gedankliche Verbindung zwischen dem umfassenden Qualitätsmanagement und Ihrem Arbeitsalltag herstellen. Das ist ein Lernprozess, der oftmals durch alte Glaubenssätze behindert wird und für alle schwierig ist. Nur durch die konsequente und ungeschönte Betrachtung der Abläufe mit den Augen der „Kundinnen“ wird die Lücke zwischen Qualitätsmanagement, Kommunikation und fachlicher Kompetenz sichtbar. Setzten Sie sich für einige Zeit die „Kundinnenbrille“ auf, befragen Sie die Frauen und Familien. Die Antworten werden überzeugen und motivieren das Team.

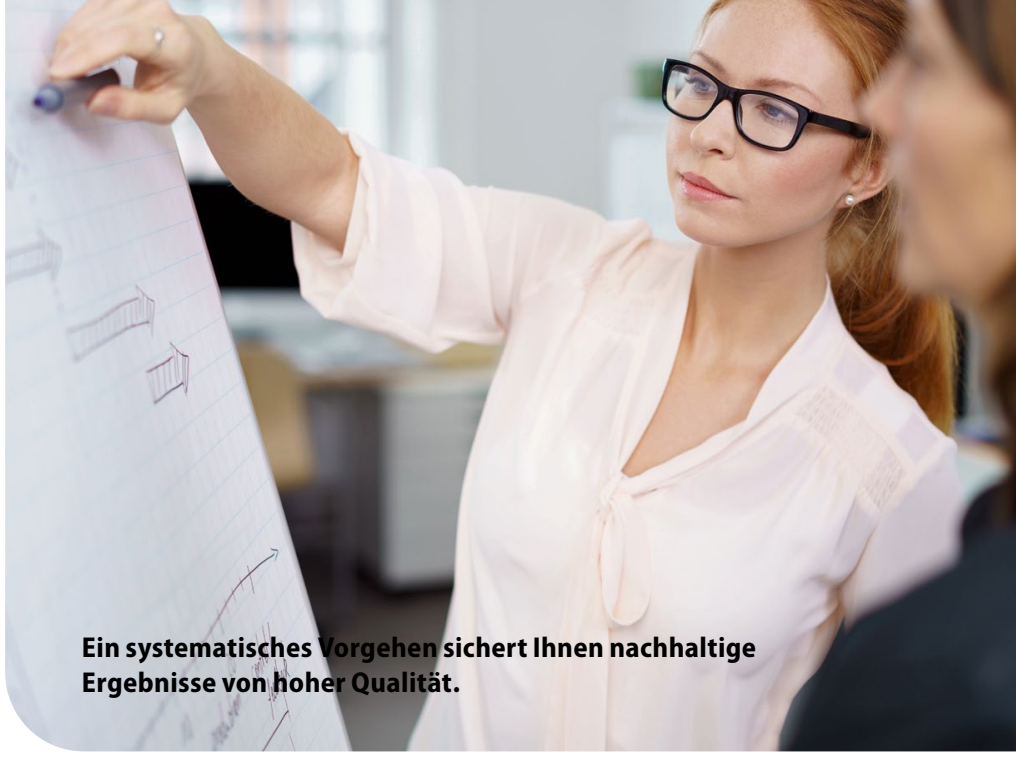

\section{Moderne Managementmethoden - auch für Hebammen}

Sie haben die Kundenanforderungen beispielsweise durch Fragebögen, Online Umfragen, Selbsteinschätzungen der Kolleginnen, Kooperationspartner aus anderen Berufsgruppen, der Literatur oder vorhandene Evidenzen ermittelt? Dann übertragen Sie die Vorschläge, die sich vielleicht auf Ihre Hebammenleistungen, Kurse, Wochenbettbetreuung, die Praxisausstattung oder die Organisation Ihrer Arbeit beziehen, auf ein großes Blatt Papier. Listen Sie die Themen als Spalten untereinander auf. Jede dieser ermittelten Verbesserungen wird vom Team mit einer Punktzahl von 1 bis 5 gewichtet. Die so gewichteten Themen sind zur „Stimme der Kundin" geworden. Allen ist nun klar, was und in welcher Reihenfolge umgesetzt wird, aber auch was nicht weiter verfolgt wird.

Erinnern Sie sich noch an die Fragen? Die von Ihnen formulierten Antworten sind Ihre Qualitätsmerkmale, das „Wie“ es sein soll. Nun stellen Sie das Wie dem Was gegenüber. Das „Was“ (die priorisierten Verbesserungen) wird vom „Wie“ (der Ausprägung der Qualität) sorgfältig getrennt. Die nun für alle Teammitglieder sichtbaren Merkmale werden verglichen und es wird beurteilt, ob sich zwei Merkmale gegenseitig unterstützen (z.B. ergänzen sich Freundlichkeit und die Akzeptanz der Eltern für ungewohnte Maßnahmen) oder sich widersprechen (z.B. konkurrieren Parkplätze vor dem Haus und eine Freifläche als Haltezone für den Rettungswagen vor der Hebammenpraxis). Wenn man nicht auf eines der beiden Merkmale verzichten kann, werden Sie diese Widersprüche mit Kreativität überwinden. Am Ende des Prozesses haben Sie den zu erwartenden Aufwand für die Umsetzung der Verbesserungsmaßnahmen schwarz auf weiß und können die einzelnen Arbeitsschritte gut planen und den Aufwand einschätzen.

\section{Die Ressourcen sind endlich}

Oft begegnen sich scheinbar gegensätzliche Ziele. Einer zunehmenden Ökonomisierung steht die Forderung nach mehr Zeit und Zuwendung für die jungen Familien gegenüber. Die aktuellen Herausforderungen für den Hebammenberuf sprechen dafür, dass Wettbewerb von gesetzgeberischer Seite erwünscht ist. Im Gesundheitswesen wird der Wettbewerb über die Offenlegung von Ergebnissen in Form von Qualitätsberichten, Zertifizierungen und QM sowie der Ausrichtung an wirtschaftlichen Prinzipien geführt. Dem kann die Hebammen nichts abgewinnen, denn das ausschließliche Bemühen um die Frauen ist noch leitend. Aber auch deren Souveränität, Aufgeklärtheit, Kritikfähigkeit und Entscheidungsfreude 


\section{$\checkmark$ PLANEN SIE REALISTISCH}

Planen Sie den Fähigkeiten und Fertigkeiten Ihres Teams entsprechend. Die folgenden 10 Punkte sind für die Durchführung Ihrer gemeinsamen Qualitätsverbesserungsprojekte hilfreich:

$\checkmark$ Gehen Sie die wichtigen Verbesserungen, die den schnellen Erfolg versprechen, zuerst an

$\checkmark$ Geben Sie dem gesamten Team viel Zeit und berichten regelmäßig über den Stand der Dinge

$\checkmark$ Erheben Sie sorgfältig den tatsächlichen Bedarf vor dem Beschluss von neuen Maßnahmen

$\checkmark$ Interessieren Sie sich für die „versteckten Kundinnenwünsche", die Sie bisher nur ahnten

$\checkmark$ Bewerten Sie die Wünsche und Anforderungen durch die "Kundinnen" nicht

$\checkmark$ Sammeln Sie alle Ideen und bewerten Sie nicht

$\checkmark$ Beschreiben Sie die Ziele genau und legen die Merkmale, an denen Sie sehen können, wie das Ziel erreicht ist, präzise fest

$\checkmark$ Legen Sie die einzelnen Schritte, die zur Verbesserung führen, vorher fest

$\checkmark$ Achten Sie auf mindestens ein Risiko, das sich aus Ihren Verbesserungsmaßnahmen ergibt

$\checkmark$ Erarbeiten Sie einen realistischen Zeitplan für die Umsetzung aller Maßnahmen

wächst, es wird bei der Freundin oder den digitalen Portalen nachgefragt und die Hebamme erfährt nachgewiesenermaßen einen Bedeutungsverlust (Rheingold Studie 2002).

\section{Der Qualitätsentwicklungsprozess nutzt unserem Team}

Ein Vorteil des systematischen Vorgehens ist, dass Sie nachhaltige Ergebnisse von hoher Qualität erhalten. Schwierige Anforderungen und Zielkonflikte werden frühzeitig identifiziert und Sie können so Ihre Ressourcen sinnvoller einsetzen. Unter dem Strich ist mit einer Beschleunigung der Umsetzung und einer Kostenreduktion zu rechnen. Alle Teamkolleginnen sind integriert und die $\mathrm{Zu}-$ sammenarbeit und Motivation verbessert sich zusehends. Zusammenhänge werden transparent dokumentiert, Entscheidungen sind nachvollziehbar und alle im Team übernehmen Verantwortung.

Die „Kundinnenwünsche “ werden nun wertneutral betrachtet und nicht mehr als Kritik an der Person. Diese sachliche Sichtweise auf die „Kundinnen/Eltern“ ohne ideologische Verzerrung dient auch der Risikominimierung. Die genaue Kenntnis des „Was“ wird von den Lösungsvorschlägen (WIE) strikt getrennt. So verhindern Sie „Schnellschüsse“, die keinen nachhaltigen Gewinn bringen und das Team letztlich demotivieren. Durch die Dokumentation der einzelnen Erarbeitungsschritte und die Einbeziehung aller Hebammen lebt Ihr Qualitätsmanagement. Sie sind zum Kern des Quali- tätsmanagements vorgestoßen, dem ständigen Verbesserungsprozess, der Planen-Durchführen-Checken-Anpassen (P-D-C-A) Methode. Die protokollierten Arbeitstreffen geben Sinn und Orientierung. Die beschlossenen Maßnahmen sind für alle nachvollziehbar und werden mit Überzeugung umgesetzt. Die Kernaufgabe, das medizinisch Notwendige und Sinnvolle zu tun, um die gesundheitliche Verfassung von Mutter und Kind zu erhalten, steht immer im Mittelpunkt.

III

\section{(1) FAZIT}

Wenn das Team Ihrer Praxis in ein Motivationsloch fällt, starten Sie eine strukturierte Qualitätsoffensive. Nehmen Sie jeden einzelnen Arbeitsprozess unter die Lupe.

Alle Teammitglieder müssen von der Sinnhaftigkeit und Notwendigkeit der gemeinsamen Vision überzeugt sein und sich für sie begeistern.

Alle Teammitglieder werden nur dann bereit sein, sich aktiv an der Festlegung der Qualitätsziele zu beteiligen, wenn sie wissen, dass Fehler als wertvoller Ausgangspunkt für Verbesserungs- und Lernprozesse betrachtet werden.

\section{Schlüsselwörter: Qualitätsmanagement, Team, Dienstleistung}

\section{Literatur}

_ Dietrich M (2005) Qualität, Wirtschaftlichkeit und Erfolg von Krankenhäusern: Analyse der Relevanz marktorientierter Ansätze im Krankenhausmanagement. DUV, Wiesbaden

_ Mayer AG (2005) Marktorientierung im Krankenhaus der Zukunft: Erfolgsfaktoren für unternehmerisch geführte Kliniken. Baumann, Kulmbach

- Saatweber J (2007) Kundenorientierung durch Quality Function Deployment. Systematisches Entwickeln von Produkten und Dienstleistungen. 2. überarb. Aufl. Symposium Verlag, Düsseldorf

_ $2 \$ 135$ a Abs. 2 SGB V

_ $3 \$ 137$ Abs. 1 Nr. $5 S G B V$

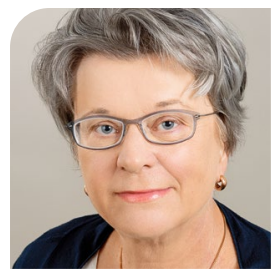

\section{Gabriele Stenz}

Qualitätsauditorin und Lehrerin für Hebammenwesen Am Hang 7 27283 Verden/Aller nds-bildung-qualitaet@gmx.net 
Hier steht eine Anzeige.

黛 Springer 